\title{
ON MINIMAL LOG DISCREPANCIES
}

\author{
FLORIN AMBRO
}

\begin{abstract}
We propose a stronger form of the boundedness of minimal log discrepancies conjectured by V.V. Shokurov. This stronger form holds up to dimension 3 and for toric varieties, and is equivalent to the lower semi-continuity of minimal log discrepancies.
\end{abstract}

\section{Introduction}

A $\log$ variety $(X, B)$ is a normal variety $X$ equipped with an effective $\mathbb{R}$ divisor $B$ such that $K+B$ is $\mathbb{R}$-Cartier, where $K$ is the canonical divisor of $X$. For any Grothendieck point $\eta \in X$, one defines the minimal log discrepancy of $(X, B)$ at $\eta$, denoted $a(\eta ; B)$ [Sh88]. This is an invariant of the singularity of $X$ at $\eta$, and is either $-\infty$, or a real number. For instance, $a(\eta ; B)=\operatorname{codim} \eta$ if $B=0$ and $X$ is nonsingular at $\eta$. The main result of this paper is the following:

Theorem 0.1. Let $(X, B)$ be a log variety. Assume that either $X$ is a toric variety and $B$ is an invariant $\mathbb{R}$-divisor, or $\operatorname{dim} X \leq 3$. If $\eta, \xi \in X$ are two Grothendieck points such that $\eta \in \bar{\xi}$, then

$$
a(\eta ; B) \leq a(\xi ; B)+\operatorname{codim}(\eta, \xi) .
$$

This motivates us to propose the following:

Conjecture 0.2. Let $(X, B)$ be a log variety. If $\eta, \xi \in X$ are two Grothendieck points such that $\eta \in \bar{\xi}$, then $a(\eta ; B) \leq a(\xi ; B)+\operatorname{codim}(\eta, \xi)$.

We also introduce new invariants of log pairs, the mld-spectrum and the mldstratification; we show that the former is a finite set and the latter is constructible (Theorem 2.3). Consequently, we obtain an equivalence between Conjecture 0.2 and Conjecture 2.4, on the lower semi-continuity of minimal log discrepancies. Roughly, the latter states that minimal log discrepancies can only decrease in special points (for instance, if $(X, 0)$ is the germ of a surface Du Val singularity, then the minimal $\log$ discrepancy at every closed point of $X$ is 2 , except at 0 , where it drops to 1 ).

The particular case $\xi=\eta_{X}$ of Conjecture 0.2 is the boundedness of minimal $\log$ discrepancies conjectured by V.V.Shokurov [Sh88], that is $a(\eta ; B) \leq \operatorname{codim} \eta$ for every Grothendieck point $\eta$ in $X$. It is known in fact that boundedness holds

Received August 11, 1999. Revised September 24, 1999.

1991 Mathematics Subject Classification. Primary: 14B05.

This work was partially supported by NSF Grant DMS-9800807. 
in the cases covered by Theorem 0.1 (cf., [Rd80, Mrk96, Ka93, Br97]), but we hope that our equivalent conjectures 0.2 and 2.4 will shed light on its general case.

\section{Acknowledgments}

I am grateful to Professor Vyacheslav V. Shokurov for useful discussions and criticism. I would also like to thank Professor Yujiro Kawamata for useful remarks.

\section{Preliminary}

A variety is a reduced irreducible scheme of finite type over a fixed field $k$, of characteristic 0 . We denote by $\eta_{X}$ the generic point of a variety $X$. A Grothendieck point $\eta \in X$ is called proper if $\eta \neq \eta_{X}$. A neighborhood of $\eta$ in $X$ is an open subset $U \subseteq X$ such that $\eta \in U$. An extraction is a proper birational morphism of normal varieties.

A log pair $(X, B)$ is a normal variety $X$ equipped with an $\mathbb{R}$-Weil divisor $B$ such that $K+B$ is $\mathbb{R}$-Cartier. $(X, B)$ is called a log variety if moreover, $B$ is effective. A log pair $(X, B)$ has log nonsingular support if $X$ is nonsingular and $\operatorname{Supp}(B)$ is a divisor with normal crossings [KMM, 0-2-9]. A log resolution of a log pair $(X, B)$ is an extraction $\mu: \tilde{X} \rightarrow X$ such that $\tilde{X}$ is nonsingular and $\operatorname{Supp}\left(\mu^{-1}(B)\right) \cup \operatorname{Exc}(\mu)$ is a divisor with normal crossings.

If $(X, B)$ is a $\log$ pair and $\mu: \tilde{X} \rightarrow X$ is an extraction, the $\log$ codiscrepancy divisor of $(X, B)$ on $\tilde{X}$ is the unique divisor $\tilde{B}$ on $\tilde{X}$ such that $\mu^{*}(K+B)=K_{\tilde{X}}+$ $\tilde{B}$ and $\tilde{B}=\mu^{-1} B$ on $\tilde{X} \backslash \operatorname{Exc}(\mu)$. The identity $\tilde{B}=\sum_{E \subset \tilde{X}}(1-a(E ; X, B)) E$ associates to each prime divisor $E$ of $\tilde{X}$ a real number $a(E ; X, B)$, called the $\log$ discrepancy of $E$ with respect to $(X, B)$. The invariant $a(E ; X, B)$ depends only on the valuation defined by $E$ on the field of rational functions of $X$, with center $c_{X}(E)=\mu(E)$. For simplicity, we write $a(E ; B)$ for $a(E ; X, B)$.

Definition 1.1. [Sh88] The minimal log discrepancy of a log pair $(X, B)$ at a proper Grothendieck point $\eta \in X$ is defined as

$$
a(\eta ; X, B)=\inf _{c_{X}(E)=\eta} a(E ; X, B),
$$

where the infimum is taken after all prime divisors on extractions of $X$ having $\eta$ as a center on $X$. We set by definition $a\left(\eta_{X} ; X, B\right)=0$.

The log pair $(X, B)$ has only log canonical (Kawamata log terminal) singularities if $a(\eta ; B) \geq 0(a(\eta ; B)>0)$ for every proper point $\eta \in X .(X, B)$ has only canonical (terminal) singularities if $a(\eta ; B) \geq 1(a(\eta ; B)>1)$ for every point $\eta \in X$ of codimension at least 2 .

Minimal log discrepancies on a log pair $(X, B)$ are computed as follows (cf., $[\operatorname{Ko} 92,17.1 .1])$. If $\operatorname{codim} \eta=1$, then $a(\eta ; B)=1-b_{\eta}$, where $b_{\eta}$ is the coefficient of $B$ in $\bar{\eta}$. Assume now $\operatorname{codim} \eta \geq 2$, and let $(\tilde{X}, \tilde{B})$ be a $\log$ resolution with a normal crossings divisor $\sum_{i} E_{i}$ supporting the divisors $\mu^{-1}(\bar{\eta})$ and $\tilde{B}$. If $(X, B)$ 
has only $\log$ canonical singularities in some neighborhood of $\eta$, then $a(\eta ; B)=$ $\min _{c_{X}\left(E_{i}\right)=\eta} a\left(E_{i} ; B\right) \in \mathbb{R}_{\geq 0}$. Otherwise, $a(\eta ; B)=-\infty$.

Example 1.2. Under the same assumptions, $a(\eta ; B)=-\infty$ if $\eta \in E$ is a proper point of a prime divisor $E$ with $a\left(\eta_{E} ; B\right)<0$.

Example 1.3. Assume that $(X, B)$ is a log pair with log nonsingular support, having only $\log$ canonical singularities at $\eta \in X$. Then $a(\eta ; B)$ is attained on the exceptional divisor of the blow-up of $X$ in $\eta$, that is

$$
a(\eta ; B)=\operatorname{codim} \eta-\operatorname{mult}_{\eta} B .
$$

Lemma 1.4. [Sh91] Assume $(X, B)$ is a log variety, and $X$ is nonsingular at $\eta$. Then $a(\eta ; B) \leq \operatorname{codim} \eta$. Moreover, $a(\eta ; B) \geq \operatorname{codim} \eta-1{\text { iff } \text { mult }_{\eta} B \leq 1}$ and $a(\eta ; B)=\operatorname{codim} \eta-\operatorname{mult}_{\eta} B$.

\section{The mld stratification}

Proposition 2.1. Assume $W \subset X$ is a closed irreducible subvariety and $(X, B)$ is a log pair with only log canonical singularities at $\eta_{W}$. Then there exists an open subset $U$ of $X$ such that $U \cap W \neq \emptyset$ and

$$
a(x ; B)=a\left(\eta_{W} ; B\right)+\operatorname{dim} W
$$

for every closed point $x \in W \cap U$.

Proof. Shrinking $X$ near $W$, we may assume there exists a log resolution $\mu$ : $(\tilde{X}, \tilde{B}) \rightarrow(X, B)$ with a normal crossings divisor $\cup_{i \in I} E_{i}$ on $\tilde{X}$ supporting $\tilde{B}=$ $\sum_{i}\left(1-a_{i}\right) E_{i}$ such that the following are satisfied:

i) $\mu^{-1}(W)=\bigcup_{i \in I_{W}} E_{i}$ for some subset $I_{W} \subseteq I$;

ii) $W \subseteq \mu(C)$ for each strata $C$ of $\cup_{i \in I} E_{i}$;

iii) $\operatorname{codim}\left(C \cap \mu^{-1}(x), C\right)=\operatorname{dim} W$ for every strata $C$ of $\cup_{i \in I} E_{i}$ dominating $W$, and every closed point $x \in W$.

iv) $(X, B)$ has only $\log$ canonical singularities and $\operatorname{dim} W>0$.

Note that $a\left(\eta_{W} ; B\right)=\min _{i \in I_{W}} a_{i}$. Fix $x \in W$. Let $\eta \in \mu^{-1}(x)$. By $\left.i i i\right)$, $a(\eta ; \tilde{B})=\sum_{\eta \in E_{i}} a_{i}+\operatorname{dim} W+\operatorname{codim}\left(\eta, C \cap \mu^{-1}(x)\right)$, where $C$ is the minimal strata of $\cup_{i \in I} E_{i}$ containing $\eta$. Since all the $a_{i}$ 's are non-negative, $a(\eta ; \tilde{B}) \geq$ $a\left(\eta_{W} ; B\right)+\operatorname{dim} W$. Taking infimum after all $\eta$ 's as above, we obtain $a(x ; B) \geq$ $a\left(\eta_{W} ; B\right)+\operatorname{dim} W$.

Finally, let $k \in I_{W}$ be an index such that $a\left(\eta_{W} ; B\right)=a_{k}$. Let $\eta$ be the generic point of an irreducible component of $E_{k} \cap \mu^{-1}(x)$ of maximal dimension: $\operatorname{codim} \eta=\operatorname{dim} W+1$. By $i i i)$ again, $E_{k}$ is the minimal strata of $\cup_{i \in I} E_{i}$ containing $\eta$. Therefore $a(\eta ; \tilde{B})=a_{k}+\operatorname{codim} \eta-1=a\left(\eta_{W} ; B\right)+\operatorname{dim} W$, and $a(x ; B) \leq a\left(\eta_{W} ; B\right)+\operatorname{dim} W$.

Definition 2.2. Let $(X, B)$ be a log pair. The mld-spectrum of $(X, B)$ is defined as the set $\operatorname{Mld}(X, B):=\{a(\eta ; B) ; \eta \in X\} \subset\{-\infty\} \cup \mathbb{R}$. We denote by $a^{o}$ the map $X \rightarrow \operatorname{Mld}(X ; B)(x \mapsto a(x ; B))$, defined on the closed points of $X$. The 
partition of $X$ into the fibers of the map $a^{o}$ is called the mld-stratification of $(X, B)$.

Theorem 2.3. Given a log pair $(X, B)$, the mld-spectrum $\operatorname{Mld}(X, B)$ is a finite set, and the mld-stratification is constructible, i.e., all the fibers of the map $a^{o}$ are constructible sets.

Proof. Suffices to prove that $\left.a^{o}\right|_{W}$ takes a finite number of values and its fibers are constructible subsets, for every closed subset $W \subseteq X$. There is nothing to prove if $\operatorname{dim} W=0$, so let $\operatorname{dim} W>0$. Let $W_{0}$ be an irreducible components of $W$. From Example 1.2 and Proposition 2.1, there exists an open subset $U_{0} \subset X$ such that $U_{0} \cap W_{0} \neq \emptyset,\left.a^{o}\right|_{U_{0} \cap W_{0}}$ is constant, and $U_{0}$ does not intersect the other irreducible components of $W$. Since $W=\left(W \backslash U_{0}\right) \sqcup\left(W_{0} \cap U_{0}\right)$, we are done by Noetherian induction.

Conjecture 2.4. [Am99] For any log variety $(X, B)$, the function $a^{o}$ is lower semi-continuous. That is, every closed point $x \in X$ has a neighborhood $x \in U \subseteq$ $X$ such that $a(x ; B)=\inf _{x^{\prime} \in U} a\left(x^{\prime} ; B\right)$.

Proposition 2.5. The two conjectures 2.4 and 0.2 are equivalent.

Proof. Assume Conjecture 0.2 is valid, and let $x \in X$ be a closed point. By Theorem 2.3, we may shrink $X$ such that $x \in \bar{C}$ for every irreducible component $C$ of the fibers of the map $a^{o}$. For $x^{\prime} \in X$, there exists a $C$ such that $x^{\prime} \in C$. Since $x \in \bar{C}$, we infer that $a(x ; B) \leq a\left(\eta_{C} ; B\right)+\operatorname{dim} \eta_{C}$. But $a\left(\eta_{C} ; B\right)+\operatorname{dim} \eta_{C}=$ $a\left(x^{\prime} ; B\right)$, so we are done.

Assume Conjecture 2.4 is valid. According to Proposition 2.1, we may assume that $\eta=\{x\}$ is a closed point and $x \in \bar{\xi}$. Let $U_{x}$ be a neighborhood of $x$ such that $a(x ; B) \leq a\left(x^{\prime} ; B\right)$ for all $x^{\prime} \in U_{x}$. Then $U_{x} \cap \bar{\xi} \subset \bar{\xi}$ is an open dense subset. From Proposition 2.1, there exists some $x^{\prime} \in U_{x} \cap \bar{\xi}$ such that $a\left(x^{\prime} ; B\right)=a(\xi ; B)+\operatorname{dim} \xi$. Therefore $a(x ; B) \leq a(\xi ; B)+\operatorname{dim} \xi$.

Remark 2.6. (V.V. Shokurov) Conjecture 2.4 is equivalent to the following lower semi-continuity in Grothendieck points: if $(X, B)$ is a log variety, every Grothendieck point $\eta \in X$ has a neighborhood $U$ such that $a(\xi ; B) \geq a(\eta ; B)$ for every Grothendieck point $\xi \in U$ with $\operatorname{dim} \xi \leq \operatorname{dim} \eta$.

Remark 2.7. If $\left(X, B_{X}\right)$ and $\left(Y, B_{Y}\right)$ are two log pairs, we denote by $(X \times$ $\left.Y, B_{X \times Y}\right)$ the product $\log$ pair, i.e., the usual product with canonical Weil divisor $K_{X \times Y}=K_{X} \times Y+X \times K_{Y}$ and pseudoboundary $B_{X \times Y}=B_{X} \times Y+X \times B_{Y}$. Then $a\left(\eta \times \xi ; B_{X \times Y}\right)=a\left(\eta ; B_{X}\right)+a\left(\xi ; B_{Y}\right)$ for Grothendieck points $\eta$ and $\xi$ on $X$ and $Y$ respectively. In particular, $\operatorname{Mld}\left(X \times Y, B_{X \times Y}\right)=\operatorname{Mld}\left(X, B_{X}\right)+$ $\operatorname{Mld}\left(Y, B_{Y}\right)$. 


\section{Conjecture 0.2 up to codimension 3}

We denote by $\mathcal{H}_{c}$ the Conjecture 0.2 with the extra assumption $\operatorname{codim} \eta=c$. Since minimal log discrepancies are preserved by passing to generic hyperplane sections, once $\mathcal{H}_{c}$ is valid for $c<d, \mathcal{H}_{d}$ is equivalent to the following particular case: if $x$ is a closed point on the $\log$ variety $(X, B)$ of dimension $d$, and $C$ is a curve passing through $x$, then $a(x ; B) \leq a\left(\eta_{C} ; B\right)+1$.

We fix $x$, and shrink $X$ to neighborhoods of $x$ without further notice. We may assume $a(x ; B)>1$, which implies that $(X, B)$ has only log canonical singularities (note that $(X, B)$ might not be Kawamata log terminal).

Theorem 3.1. $\mathcal{H}_{c}$ is valid for $c=1,2,3$.

Proof. $\mathcal{H}_{1}$ can be easily checked on curves. For $\mathcal{H}_{2}$, let $x \in C \subset X$ be as above, with $\operatorname{dim} X=2$. Since $a(x ; B)>1, X$ is nonsingular at $x$ and $a(x ; B)=$ $2-\operatorname{mult}_{x} B$. In particular, $a(x ; B)-\left(a\left(\eta_{C} ; B\right)+1\right)=\operatorname{mult}_{C} B-\operatorname{mult}_{x} B \leq 0$.

For $\mathcal{H}_{3}$, let $x \in C \subset X$ be as above, with $\operatorname{dim} X=3$. Assume first $a\left(\eta_{C} ; B\right) \leq 1$. From the Log Minimal Model Program (cf., [Ka92]), there exists a crepant extraction $\mu:(\tilde{X}, \tilde{B}) \rightarrow(X, B)$ such that $\tilde{B}$ is effective and there exists a prime divisor $E$ on $\tilde{X}$ with $\mu(E)=C$ and $a\left(\eta_{E} ; \tilde{B}\right)=a\left(\eta_{C} ; B\right)$. Let $\eta$ be the generic point of a curve in the fiber of $\left.\mu\right|_{E}: E \rightarrow C$ over $x$. By $\mathcal{H}_{2}$, $a(x ; B) \leq a(\eta ; \tilde{B}) \leq a\left(\eta_{E} ; \tilde{B}\right)+1$.

Let now $a\left(\eta_{C} ; B\right)>1$. We may assume that $a(x ; B)>2$. By Lemmas 3.2 and 1.4, $X$ is nonsingular at both $x$ and $\eta_{C}$ and $a(x ; B)-\left(a\left(\eta_{C} ; B\right)+1\right)=$ $\operatorname{mult}_{C} B-\operatorname{mult}_{x} B \leq 0$.

Lemma 3.2. Let $x$ be a closed point on a log variety $(X, B)$ of dimension 3. Then $X$ is nonsingular point at $x$ if $a(x ; B)>2$.

Proof. We first show that $X$ has $\mathbb{Q}$-factorial singularities. Indeed, from the Log Minimal Model Program we can find a $\mathbb{Q}$-factorialization $\mu:(\tilde{X}, \tilde{B}) \rightarrow(X, B)$, where $(\tilde{X}, \tilde{B})$ is a $\log$ variety again. Since $a(x ; B)=\min _{\eta \in \mu^{-1}(x)} a(\eta ; \tilde{B})$, we infer from $\mathcal{H}_{1}$ and $\mathcal{H}_{2}$ that $\operatorname{dim} \mu^{-1}(x)=0$. Zariski's Main Theorem (cf., [Ha77, Exercises II.3.22, III.11.2]) implies that $\mu$ is an isomorphism over a neighborhood of $x$, hence $X$ is $\mathbb{Q}$-factorial.

From the proof of Theorem 3.1, $a\left(\eta_{C} ; B\right)>1$ for every curve passing through $x$. By $\mathcal{H}_{2},(X, B)$ and $X$ have only terminal singularities. If $x$ is a singular point of $X$, then it is an isolated terminal singularity, hence $a(x ; B) \leq a(x ; 0)=1+\frac{1}{r} \leq$ 2, where $r$ is the index of $K_{X}$ at $x$ [Rd80, Mrk96, Ka93]. Contradiction!

The following characterization of cDV singularities is folklore. We include a sketch of its proof for completeness:

Proposition 3.3. Assume $(X, B)$ is a log variety of dimension 3 and let $x \in X$ be a closed point. Then $a(x ; B)=2$ iff exactly one of the following holds:

i) $X$ is nonsingular at $x$ and mult $_{x} B=1$.

ii) $x \notin \operatorname{Supp}(B)$ and $X$ has a $c D V$ singularity at $x$ (cf., $[\operatorname{Rd} 80])$. 
Sketch of proof. By Lemma 1.4 we may assume that $x$ is a singular point of $X$. By lower semi-continuity, $(X, B)$ has only canonical singularities. Assume first that $B$ is $\mathbb{R}$-Cartier. By Lemma $3.2, B=0$ near $x$. According to [Rd80, 2.2], suffices to show that $K_{X}$ is a Cartier divisor. If $X$ has terminal singularities at $x$, then $K_{X}$ is Cartier by [Rd80, Mrk96, Ka93]. Otherwise, let $\mu: \tilde{X} \rightarrow X$ be an extraction such that $\mu^{*} K_{X}=K_{\tilde{X}}$ and $\tilde{X}$ has terminal singularities ([Rd83, 0.6]). The terminal subcase implies that $K_{\tilde{X}}$ is Cartier near $\mu^{-1}(x)$, thus $K_{X}$ is Cartier by the Contraction Theorem.

Assume now that $B$ is not $\mathbb{R}$-Cartier. Let $\mu:(\tilde{X}, \tilde{B}) \rightarrow(X, B)$ be a small extraction such that $\tilde{X}$ is $\mathbb{Q}$-factorial. Then $\mu^{-1}(x)$ is a union of curves, none of them included in the support of $\tilde{B}$. In particular, $-K_{\tilde{X}}$ is $\mu$-nef, but not $\mu$-trivial. However, $\tilde{X}$ admits no flipping contraction: its difficulty [Sh86] is 0 since it has only terminal Gorenstein singularities. Contradiction!

\section{Toric minimal log discrepancies}

We refer the reader to [Fu93] for definitions and basic notations of toric geometry. Let $X=T_{N} \operatorname{emb}(\Delta)$ be a toroidal embedding, and let $\left\{B_{i}\right\}_{i=1}^{r}$ be the $T_{N}$-invariant divisors of $X$, corresponding to the primitive vectors $\left\{v_{i}\right\}_{i=1}^{r}$ on the 1-dimensional faces of $\Delta$. Let $B=\sum_{i}\left(1-a_{i}\right) B_{i}$ be an invariant $\mathbb{R}$-divisor such that $K+B$ is $\mathbb{R}$-Cartier. Equivalently, there exists a linear form $\varphi \in M_{\mathbb{R}}$ such that $\varphi\left(v_{i}\right)=a_{i}$ for every $i$. We may assume the $\log$ variety $(X, B)$ has only $\log$ canonical singularities, i.e., $0 \leq a_{i} \leq 1$ for every $i$.

Under the above assumptions, we have the following formula for the minimal $\log$ discrepancies of $(X, B)$ at the generic points of the orbits (cf., [Br97]):

$$
a_{\sigma}:=a\left(\eta_{\operatorname{orb}(\sigma)} ; B\right)=\inf \{\varphi(v) ; v \in \operatorname{rel} \operatorname{int}(\sigma) \cap N\}, \sigma \in \Delta .
$$

Here, rel $\operatorname{int}(\sigma)$ denotes the relative interior of $\sigma \subset \mathbb{R} \sigma$, and $\operatorname{orb}(\sigma)$ is the $T_{N^{-}}$ orbit corresponding to the cone $\sigma \in \Delta$. Conjecture 2.4 for toric varieties follows from the following:

Theorem 4.1. In the above notations, let $X=\bigsqcup_{\sigma \in \Delta} \operatorname{orb}(\sigma)$ be the partition of $X$ into $T_{N}$-orbits.

i) Each strata in the mld-stratification is a union of orbits. In other words, $a(x ; B)=a_{\sigma}+\operatorname{codim}(\sigma)$ for every cone $\sigma \in \Delta$ and every closed point $x \in \operatorname{orb}(\sigma)$.

ii) $a_{\sigma}+\operatorname{codim}(\sigma) \leq a_{\tau}+\operatorname{codim}(\tau)$ for all cones $\tau, \sigma \in \Delta$ such that $\tau$ is a face of $\sigma$ (i.e. $\operatorname{orb}(\sigma)$ is in the closure of $\operatorname{orb}(\tau))$.

Proof. $i)$ : The equality holds for the generic closed point $x \in \operatorname{orb}(\sigma)$ from Proposition 2.1. This extends to all the points in $\operatorname{orb}(\sigma)$ since $T_{N}$ acts transitively on orbits and leaves the boundary fixed.

ii) : Let $\tau$ be a proper face of $\sigma$ and let $a_{\tau}=\varphi(v)$ for some $v \in \operatorname{rel} \operatorname{int}(\tau) \cap N$. There exist primitive vectors $v_{i_{1}}, \ldots, v_{i_{c}}(c=\operatorname{codim}(\tau, \sigma))$ on the 1-dimensional faces of $\sigma$ such that $w=v+v_{i_{1}}+\cdots+v_{i_{c}} \in \operatorname{rel} \operatorname{int}(\sigma)$. Therefore $a_{\sigma} \leq \varphi(w)=$ $\varphi(v)+a_{i_{1}}+\cdots+a_{i_{c}} \leq a_{\tau}+\operatorname{codim}(\tau, \sigma)$. 
V.V. Shokurov also conjectured the following nonsingularity criterion [Sh88]: if $(X, B)$ is a $\log$ variety and $a(\eta ; B)>\operatorname{codim} \eta-1$, then $X$ is nonsingular at $\eta$. If $X$ is a toric variety and $B$ is an invariant $\mathbb{R}$-divisor, this holds due to the following:

Proposition 4.2. Let $\sigma \subset N_{\mathbb{R}}$ be a strongly rational polyhedral cone generated by the primitive vectors $v_{1}, \ldots, v_{r} \in N$. Assume $\varphi \in M_{\mathbb{R}}$ is a linear form such that $0 \leq \varphi\left(v_{i}\right) \leq 1$ for every $i$, and let

$$
\varphi_{\sigma}:=\inf \{\varphi(v) ; v \in \operatorname{rel} \operatorname{int}(\sigma) \cap N\} .
$$

If $\varphi_{\sigma}>\operatorname{dim} \sigma-1$ then $\sigma$ is a nonsingular cone.

Sketch of proof. We use induction on $n=\operatorname{dim} \sigma$. By lower semi-continuity, $\varphi$ has the same property when restricted to any proper face of $\sigma$. In particular, every proper face of $\sigma$ is nonsingular.

If $\sigma$ is simplicial, i.e., $r=n, \varphi_{\sigma} \leq \frac{n}{2}$ unless $\sigma$ is a nonsingular cone (cf., [Br97]). If $\sigma$ is not simplicial, one may assume $r=n+1$. This implies $v_{n+1}=$ $v_{1}+\ldots+v_{s}-v_{s+1}-\ldots-v_{k}$, where $s \geq 1$ and $s+1 \leq k \leq n$. Therefore $\varphi_{\sigma} \leq \varphi\left(v_{1}+\ldots+v_{s}+v_{k+1}+\ldots+v_{n}\right) \leq s+n-k \leq n-1$. Contradiction!

\section{References}

[Am99] F. Ambro, The adjunction conjecture and its applications, PhD thesis 1999.

[Br97] A.A. Borisov, Minimal discrepancies of toric singularities, Manuscripta Math. 92 (1997), 33-45.

[Fu93] W. Fulton, Introduction to toric varieties, Annals of Mathematics Studies, 131. The William H. Roever Lectures in Geometry. Princeton University Press, Princeton, NJ, 1993.

[Ha77] Hartshorne, R., Algebraic Geometry, Graduate Texts in Mathematics, No. 52. Springer-Verlag, New York-Heidelberg, (1977).

[Ka88] Y. Kawamata, Crepant blowing-up of 3-dimensional canonical singularities and its application to degeneration of surfaces, Ann. of Math. (2), 127 (1988), 93-163.

[Ka92] , Termination of log flips for algebraic 3-folds, Internat. J. Math. 3 (1992), $653-660$.

[Ka93] , The minimal discrepancy of a 3-fold terminal singularity, An appendix to [Sh93].

[KMM] Y. Kawamata, K. Matsuda, and K. Matsuki, Introduction to the minimal model problem, Algebraic Geometry, Sendai, 1985, 283-360, Adv. Stud. in Pure Math. 10. North-Holland, Amsterdam-New York, (1987).

[Ko92] J. Kollár et al., Flips and abundance for algebraic threefolds, Astérisque 211 (1992).

[Mrk96] D. Markushevich, Minimal discrepancy for a terminal cDV singularity is 1, J. Math. Sci. Univ. Tokyo 3 (1996), 445-456.

[Mr85] S. Mori, On 3-dimensional terminal singularities, Nagoya Math. J., 98 (1985), 43-66.

[Rd80] M. Reid, Canonical 3-folds, in Géométrie algébrique Angers, 1979, (A. Beauville, ed.), 273-310, Sijthof \& Noordhoff, Alphen aan den Rijn-Germantown, Md., 1980.

[Rd83] _ Minimal models of canonical threefolds, in Algebraic varieties and analytic varieties, (Tokyo, 1981), 131-180, Adv. Stud. Pure. Math., 1, North-Holland, Amsterdam-New York, 1983.

[Sh86] V.V. Shokurov, A nonvanishing theorem, Math. USSR Izv. 26 (1986), 591-604.

[Sh88] , Problems about Fano varieties, Birational Geometry of Algebraic Varieties, Open Problems — Katata 1988, 30-32. 
[Sh91] - A.c.c. in codimension 2, preprint 1991.

[Sh93] , 3-Fold log flips, Russian Acad. Sci. Izv. Math. 40 (1993), 95-202.

Department of Mathematics, University of California, Santa Barbara, CA 93106

E-mail address: ambro@math.ucsb.edu 